\title{
Social Cognitive Neuroscience Perspective for Unification Psychology
}

\author{
Wangbing Shen, Chang Liu, Yuan Yuan \\ Lab of Cognitive Neuroscience, School of Education Science, Nanjing Normal University, \\ Nanjing, China. \\ Email: liuchang@njnu.edu.cn,won.being.shin@gmail.com \\ Received July $3^{\text {rd }}$, 2011; revised August $13^{\text {rd }}$ 2011; accepted September21 ${ }^{\text {st }}, 2011$.
}

\begin{abstract}
As a direct part of these disciplines which focuses on human's soul and mind, psychology has a history of more than one hundred years. However, there are still many people who have lots of doubts for psychology, especially on the division and integration of psychology which dates back to Spence (1987). Many studies in the past have sought for many paradigms to integrate psychology, but they failed, such as cognitive psychology paradigm. One challenge to the traditional perspective comes from works of social cognitive neuroscience. More and more specific studies showed that social cognitive neuroscience can integrate psychological science. Therefore, increasing psychologists, including theoretical psychologists focus their sights on social cognitive neuroscience. The article gave an overview analysis to those problems, including the integration from social cognitive neuroscience is possible and its prospect framework of integration.
\end{abstract}

Keywords: Social Cognitive Neuroscience, Psychology, Humanity Hypothesis, Psy-DNA Model

\section{What's Social Cognitive Neuroscience?}

Social cognitive neuroscience (SCN) dated back to the beginning of twenty-first century. Oschner and Lieberman (2001) gave a concrete interpreting for SCN. They pointed out that SCN evolved from the interdisciplinary combination between social psychology and cognitive neuroscience. Meanwhile, they also argued that its goal seemed to understand phenomena in terms of interactions across three levels, including social, cognitive and neural. In particular, the name SCN denotes the interdisciplinary nature of this field, and emphasizes the characteristic of integrating data from multiple levels ranged from individual experience and behavior in the social contexts (social level) to information processing mechanisms giving rise to those phenomena (cognitive level) to the brain base (neural level).

SCN is still relatively new; however, it builds on a lot of well established disciplines including social psychology, developmental psychology and cognitive psychology, evolutionary biology, neuropsychology and computer science. Each provides a solid basis of relevant research for SCN from different aspects (Blakemore, Winston, \& Frith, 2004). In particularly, those studies on social cognition contribute more to the development of SCN, including stereotype, attitude forming and or attitude change, social judgment, self-knowledge and the interaction between emotion and cognition. Then, the technologies such as brain imaging or event related potentials (ERPs) contribute to it. In a word, SCN encompasses numerous empirical studies on neural mechanism underlying social cognitive process (Blakemore, Winston, \& Frith, 2004), and those studies on general cognitive process such as perception, language and memory.

\section{Could SCN Integrate Psychological Science?}

When we argue that SCN will integrate different fields of psychological science, there are many researchers or studies maybe question that why would be SCN, not others, and how does SCN integrate those divided approaches. We will discuss these problems in the following. However, we have to introduce the concept and construct of humanity hypothesis which the integration depends.

\section{Humanity Hypothesis Is the Beginning of Unification}

In general, there are two approaches in psychological science. One is human science approach, the other is natural science approach. The former is mainly guided by phenomenological philosophy, whereas the latter is instructed by empirical philosophy. Just so, those two guidelines result in they are different not only in their views but also in their methods. Specifically, human science approach in psychology mainly employs qualitative paradigm. It pays more attention to unique value of person because it believes that there is important difference between person and machine such as computer, or animals such as Thorndike's cat or Tolman's rat. In contrast, the natural science approach of psychology mainly argues that the psyche or mind can be reduced to logic or processing of machine or others, such as some activities of animals. Also for this, many radical theoretical psychologists name it reductionist. Hence, there is a great gap between these two approaches in methodology and method. As many textbooks mentioned, the former investigate psychological process and cognitive mechanism mainly depending on narrative analysis or other phenomenological methods, whereas the latter do this primarily depending on quantitive measures such as lab experiment and psychological test.

According to the aforementioned, there is a great difference between these two approaches ranging from guidelines to methods. People should realize that how different they are, although they all belong to psychological science. In this sense, they all explore and try to clarify humanity. Namely, there is a consensus between them. In sum, they want to explore and discuss mind and behaviors of human being.

Unified Humanity Determines Unification Psychology

In psychology, there are numerous humanity hypotheses, 
such as "self-realization human" hypothesis and "complex human" hypothesis. Different approaches of psychology are developed based on different humanity hypotheses. For example, our humanistic psychology was developed by Maslow based on self-realization human hypothesis. If we want to investigate the reasons of division in psychological science, we will find that humanity hypothesis plays an important role in it. Why is it? Because those humanity hypotheses are so different that they guided different approaches. However, those hypotheses reach little consensus. That is, those approaches of psychology which based on different or contrary humanity hypotheses share little similarity. Hence, different humanity hypotheses, to some extent, result in the division of psychology.

When you critically reviewed those humanity hypotheses, you will find that each of them is not appropriate, because they only focuses on one side of humanity. In other words, human nature has been constructed different second-order humanities clarified by different humanity hypotheses. Second-order human nature is based on humanity on high ground, not primary and low ground, such as morality which was not based on basic and low ground, but based on high ground (similar to a noble person rather than an usual person). One prominent problem on humanity hypothesis is that it only focuses on social or psychological aspect of human nature, rather than every side of humanity. In such sense, a humanity hypothesis determines which direction psychological science goes ahead. If humanity is unified, people will perhaps have a unification psychology.

To resolve such sharp difference in the literature, it is necessary to construct a new and unified humanity hypothesis. Thus, we try to propose a unified humanity hypothesis (see Figure 1). The investigators believe that humanity includes different elements. Humanity co-evolved with sociality, physic nature, biological basis and experience or culture. Meanwhile, humanity is evolving with the growth of a person.

\section{Do Those Relate to SCN?}

Although previous content showed that humanity hypothesis seems to play a critical role in unification psychology, it has nothing with SCN. If so, why does SCN integrate psychological science? First, we should argue for SCN, because SCN relate to humanity hypothesis, and even to integration of psychology science.

The main task of psychology is exploring consciousness and mind of human being. However, our behaviors and minds are shaped by our experiences and their underlying neurophysiologic mechanisms. Among these, our experiences often occur

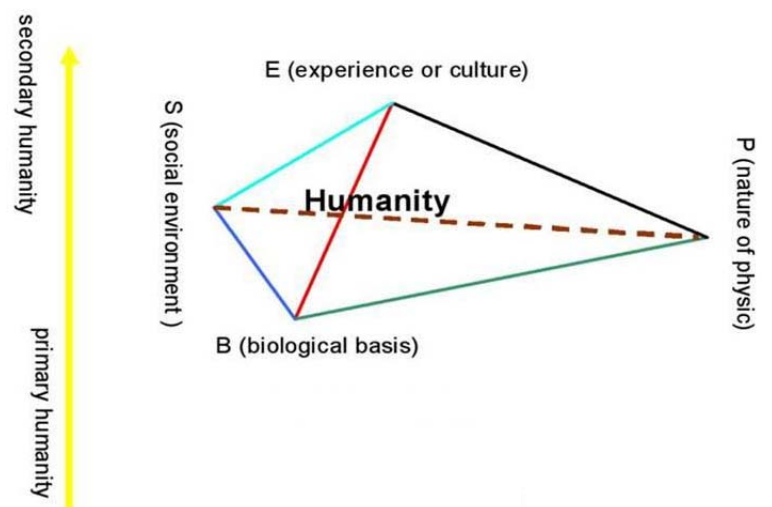

Figure 1.

Humanity hypothesis. in the context of the nurture, or mainly culture in which we develop and live, while, neurophysiologic mechanisms of our behaviors and minds mainly determined by our genes and brain, which influenced and even decided by our parents. In a word, our behaviors and minds are mainly determined by their genetic background and their experiences, thee former also named nature, the later nurture.

What we have referred, SCN combines mainly cognitive neuroscience and social psychology. However, an important goal of social neuroscience research is to understand the basic genetic and neurobiological mechanisms underlying human sociality. Although this information can then be used for the development of novel treatment strategies for clinical disorders associated with disrupted social behaviors. Unfortunately, basic and clinical human research has been severely limited in its ability to dissect the complex interactions of genes, the environment, and the neural mechanisms underlying normal social processes (Cacioppo, Amaral et al., 2007). It is not difficult to find that SCN try to understand neural basis of our behaviors and their underlying phenomena meaning. Meanwhile, we also understand these relationships between humanity and SCN. As noted above, SCN builds on a variety of well established disciplines including social, developmental and cognitive psychology, evolutionary biology, neuropsychology and computer science, especially social psychology and cognitive neuroscience. What we should emphasize, social psychologies mainly focus on social phenomena and its psychological meanings, cognitive neuroscience mainly pay attention to neural or physiological basis of minds and behaviors. However, humanity is mainly influenced by such two factors. Therefore, SCN relate to humanity hypothesis, and even to integration of psychology science.

\section{SCN Integrates Psychology: A Framework}

\section{Psy-DNA Model}

In this respective, we proposed a unification framework corresponding to our humanity hypothesizes, which named PsyDNA model and dated back to Wundt and inspired by Wason and Crick's DNA model (see Figure 2). In Wundt's time, Wundt has tried to sketch a blueprint of psychology system. His works of "Contribution to the theory of sensory perception" depict a young scientist's dream; he originally proposed a great idea that he wants to construct a discipline composed by both experiment psychology and social psychology. What we should point is that the underlying understandings of so-called experiment psychology and folk psychology, at least in following understanding of both. In classical works of psychology, Wundt's experiment psychology more has been viewed physicological psychology, which acquired their results and conclusion by experiment, including experiment in the labs or in the field. However, there are many different viewpoints or opinions focus on the understanding of Wundt's folk psychology, some studies believe in that there is not any difference between culture psychology and folk psychology, some researchers argued that the meaning of folk psychology is equal to social psychology, although more people argued for the latter. In Wundt's following works, such as "The Principles of Physiological Psychology", he has given a more specific and detailed elaboration for his science dream in its preamble. According to his clarification in "The Principles of Physiological Psychology", the folk psychology refers to the latter subject who includes more different fields, such as law, art and morality which related to 


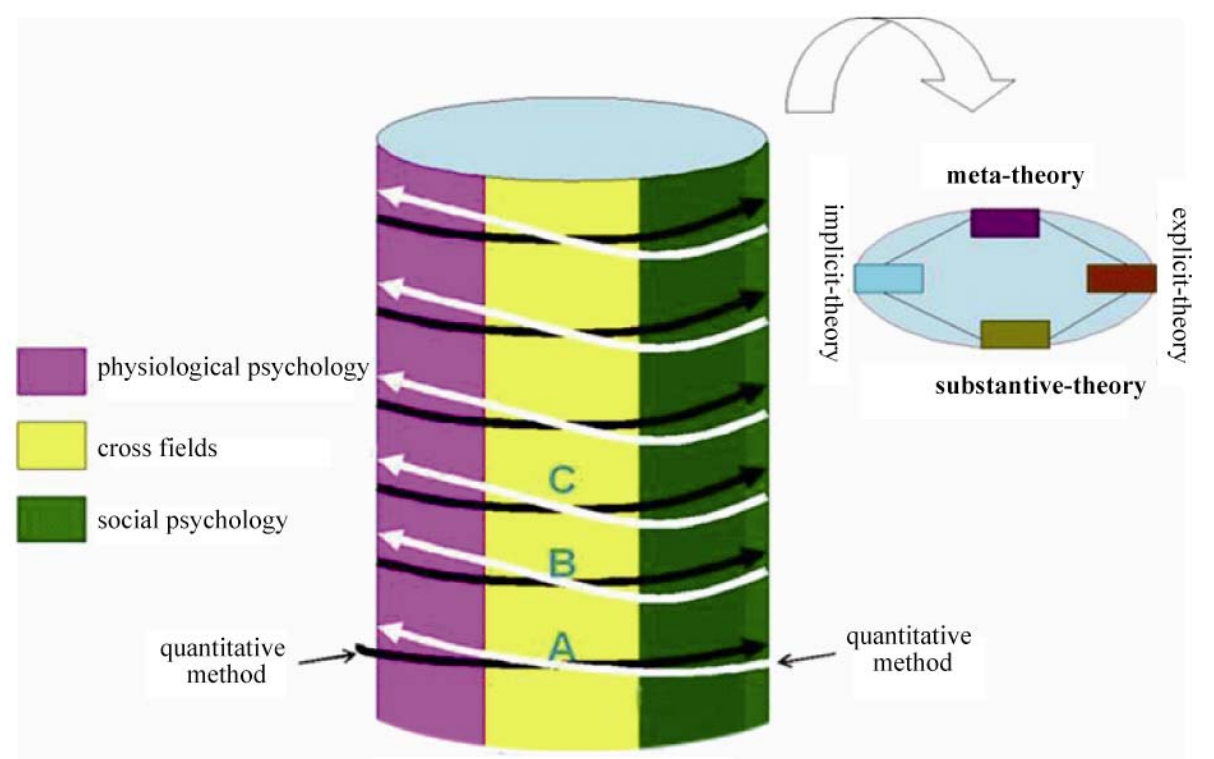

Symbols and abbreviation in Figure 2: A = autobiography analysis, $\mathrm{B}=$ simulation, $\mathrm{C}=$ meta-analysis, Psy-DNA = integrated Psychology science analogy to DNA.

Figure 2.

Psy-DNA Model.

social phenomenon. That is, the Psy-DNA model is developed from Wundt's psychology system.

Psy-DNA model outlined a framework for the study of psychology integration, currently one of the liveliest topics in the theoretical psychology. The framework has come to be known as Psychology DNA model (Psy-DNA), because it seeks to describe the means and constructions of psychology science by using concepts and models similar to those used in Wason and Crick's DNA program in biology. Psy-DNA model shared many features with others important research programs in psychology and neuroscience, particularly an emphasis on describing the interdisciplinary principle of psychology unification, Such as evolutionary neuroscience or cognitive neuroscience, even cognitive psychology. However, Psy-DNA model has some distinct characteristics that set it apart from other influential approaches, especially from biological DNA model. for example it has involved four different aspects of construct which named meta-theory, substantive theory, implicit theory and explicit theory. Among these, meta theory and substantive theory construct covariant, they focus on different aspects of psychology science, meta theory mainly focus on "theory on theory" like "cognition on cognition", however, substantive theory pay more attention to the construction of basic theory and theoretical interpreting for social phenomena and behaviors. Similarly, implicit theory and explicit theory mainly focus on distinctive aspects of some phenomenon and behavior. Specifically, implicit theory mainly concerns on implicit aspect of behavior or mind, but explicity theory pay more attention to explicit aspect. Furthermore, the double helix of DNA model as a metaphor of two kinds of method in psychology science, namely quantitative method and qualitative method. These two different method-linkages spiral change with Psy-DNA model like double helix of DNA.

\section{Initial Evidence}

Initial evidence supporting Psy-DNA model mainly comes from biology, philosophy, methodology, psychology and SCN. None of that evidence is univocal or conclusive, however, it provides at least modest support for the hypothesis social cognitive integrate psychological science.

First, various philosophy studies revealed the development of one thing does not go beyond the alternative one directly. PsyDNA model agrees with philosophical principle. Those two different approaches are contradictory, but they are a unity of opposites not only in method (quantitative vs. qualitative) but also in research paradigm (scientism vs. humanism). In PsyDNA model, two different method-linkages stand for the two different approaches, namely scientism approach and humanism approach in psychological science.

Second, many methodological studies indicated that no matter which method it is, it had disadvantages and advantages. That is, no one goes beyond the other in any way. Instead, most methods or approaches are internally modulated. Furthermore, they cross-talk and interactively influence and then reach a cross-develop. As developed psychological methods, studies revealed that different psychological methods also communicated with each other. They did not argue against each other, only reach a compromise.

Third, Psy-DNA model is supported by DNA model proposed by Watson and Crick. They pointed out that DNA structure included a double helix composed by four different types of bases. The P-DNA model also has double helix linkages. Those two linkages are psychological method and research approach, respectively. One stands for quantitative method or empirical approach, and the other stands for qualitative method or qualitative approach. However, according to analogism theory proposed by Guangjian Zhang and Xueshen Qian, one important advance not only takes an important influence in one field, but also influences its adjacent fields. That is, DNA model perhaps does not appear in biology, it or its proxy probably appears in other disciplines, such as psychology science.

Finally, more direct evidence supporting such a unification 
model come from psychology and cognitive neuroscience. Besides those supporting from Wundt's studies, there are many evidence from cognitive psychology and functional imaging researches, such as those studies on dual process of explicit and implicit processing. In social and cognitive psychology, this view has been widely accepted. Many researches showed that there are two processes play roles in decision making, social judgment and problem solving (Greene et al., 2008, 2004; Hadit, 2001; Chaiken \& Trope, 1999; Shen \& Liu, 2010). Meanwhile, they found that there are two systems underlying cognitive process, which typically run in parallel and could reach differential conclusions. Just for this, such models are usually called as dual process model. Dual process has a powerful influence on memory, arithmetic, deduction reason, moral judgment, decision-making and social evaluation. Although it comes forth as different form in different psychological process or states (i.e., attitude or personality), it shares the same characteristics. One processing is explicit and influenced by cognitive load, the other is implicit and automatic (Shen \& Liu, 2010). Similarly, there are also much evidence supporting the construct of meta-theory and substantive theory, such as metacognition and cognition.

\section{Evidence from SCN Studies}

Recently, the emerging SCN has also get many results and conclusions which consistent with Psy-DNA, Such as morality. Although morality evolve both by biological basis and social development, we often view it influenced and developed by social interaction and inter and or intra relationships. Therefore, most psychologists get an impact that morality is a social phenomenon, mainly researched by ethicists. Piaget broke out such tradition, becomes a moral psychologist. Then more and more psychology studies focus on morality by psychology methods, such as Kohlberg.

As the development of cognitive neuroscience and emerging of SCN, some scientists studied morality by functional imaging technologies, such as fMRI. Greene et al. (2001) investigated moral judgment by presenting participants moral dilemmas. In this experiment two types of dilemmas were contrasted, represented by dilemmas, such as the "trolley dilemma" and the "footbridge dilemma". In these impersonal dilemmas like trolley dilemma the participant is asked to consider the following situation: A run away trolley is quickly approaching a fork in the tracks. On the tracks extending to the right is a single railway workman. If one does nothing, the trolley will proceed to the left, causing the deaths of the five workmen. The only way to avoid the deaths of these workmen is to hit a switch on your dashboard that will cause the trolley to the right railway, but this will cause the single workman die. After presenting this story, the participant in the experiment is asked to respond whether it is appropriate to hit the switch to avoid the deaths of the five workmen. In the footbridge dilemma the situation is slightly different. Again, a run away trolley is heading down the tracks toward five workmen who will be killed if the trolley proceeds on its present course. The participant now has imagined on a footbridge being on a footbridge over the tracks and in between the approaching trolley and the five workmen. Next on the footbridge stand a large stranger. The only way to save the lives of the five workmen is to push the large stranger off the bridge and onto the tracks below. The stranger who will lose his life is so large that he can stop the trolley, but you are so thin that you can't. After presenting this situation, the participants again were asked to respond whether it is appropriate to push the stranger onto the tracks to save the five workmen. By comparing neural activity during reasoning about these different types of dilemmas, they found moral judgment is co-determined by irrational and rational processing, and the former mainly include emotion while the latter mainly include cognition. Specifically, irrational processing involved in personal dilemmas like footbridge dilemma mainly activated medial prefrontal cortex, the posterior cingulate cortex and the posterior superior temporal sulcus. However, whereas retional processing involved in personal dilemmas like trolley dilemma mainly activated the dorsolateral prefrontal cortex and inferior parietal lobe. Then Moll et al. (2001, 2002), Koenigs et al. (2007a, 2007b) and Greene (2004, 2008) have also studied the neural activity of morality. Like this, there are many studies focusing on other social cognition, such as theory of mind and other cognition, such as the neural activity of memory or arithmetic.

In sum, there is great difference between SCN and traditional cognitive neuroscience. SCN not only focuses on neural activity underlying social phenomena, but also on social meaning of it. Importantly, it build a connection between physiological basis and social phenomena, not single social phenomena like social psychology and not single physiological basis in physiological psychology or neuroscience. It maps social phenomena to its neural activity, which contacts social psychology with physiological psychology. In other word, it also maps person's social behaviors to its underlying neural basis. Besides, SCN is different for cognitive neuroscience, it not only focus on traditional "cognition", but also on emotion and other social phenomena.

\section{Conclusion Remarks}

The goal of this paper seems to discuss the framework on unification psychology at an aspect of human humanity. Although we give a blueprint of unification psychology from SCN perspective, it still needs further studies in detail.

From SCN perspective, the mechanisms underlying mind and behaviors are not fully explained by either biological or social approach rather than an interaction even multilevel integrative analysis if necessary. All human behaviors and mind that psychology investigates, to some extent, are biological. However, it does not support that biological reductionism could produce a simple, singular, or satisfactory explanation for various and complex behaviors. In other words, we do not believe that neural representation or molecular mechanism can provide a unique way or the best interpreting for understanding humanity and their behaviors (Cacioppo, Berntson, Sheridan, \& McClintock, 2000). Within those disciplines of psychology, our mind and behavior are shaped by two factors, namely social environment and biological basis. However, those specific studies are explored and fulfilled by different fields of psychological science. It implies that if people want to understand human and humanity, they need integrate those different fields, even unified psychology. In fact, physiological psychology focuses on neural substrates and brain mechanisms for behaviors, whereas social psychology emphasizes multivariate system and situational effects in studying the impact of human association on mind and behavior (Cacioppo, Berntson, Sheridan, \& McClintock, 2000). The aforementioned approaches directly result in different analytical level of human mind and behavior. In a word, people should view human mind and behavior from an integrative respective, especially for the unification perspective of social influences, physiological basis and 
their connections.

In summary, the present paper only provides a preliminary framework from SCN and some initial evidence, it is very necessary to bridge the gap between initial exploration and programmatic experiment. Thus, it is extremely important to investigate further studies in detailed.

\section{References}

Blakemore, S. J., Winston, J., \& Frith, U. (2004). Social cognitive neuroscience: Where are we heading? Trends in Cognitive Sciences, 8, 216-222. doi:10.1016/j.tics.2004.03.012

Chaiken, S., \& Trope, Y. (1999). Dual process theories in social psychology. New York: Guilford Press.

Cacioppo, J. T., Berntson, G. G, Sheridan, J. F., \& McClintock, M. K. (2000). Multilevel integrative analysis of human behavior social neuroscience and the complementing nature of social and biological approaches. Psychological Bulletin, 126, 829-843.

doi:10.1037/0033-2909.126.6.829

Cacioppo, J. T., Amaral, D. G., Blanchard, J. J., Cameron, J. L., Carter, C. S., Crews, D., et al. (2007). Social neuroscience: Progress and implications for mental health. Perspectives on Psychological Science, 2, 99-123. doi:10.1111/j.1745-6916.2007.00032.x

Greene, J. D., Sommerville, R. B., Nystrom, L. E., Darley, J. M., \& Cohen, J. D. (2001). An fMRI investigation of emotional engagement in moral judgment. Science, 293, 2105-2108. doi:10.1126/science.1062872

Greene, J. D., Nystrom, L. E., Engell, A. D., Darley, J. M., \& Cohen, J. D. (2004). The neural bases of cognitive conflict and control in moral judgment. Neuron, 44, 389-400. doi:10.1016/j.neuron.2004.09.027

Greene, J. D., Morelli, S. A., Lowenberg, K., Nystrom, L. E., Cohen, J.
D. (2008). Cognitive load selectively interferes with utilitarian moral judgment. Cognition, 107, 1144-1154. doi:10.1016/j.cognition.2007.11.004

Haidt, J. (2001). The emotional dog and its rational tail: A social intuitionist approach to moral judgment. Psychological Review, 108, 814-834. doi:10.1037/0033-295X.108.4.814

Moll, J., Eslinger, P. J., de Oliveira-Souza, R. (2001). Frontopolar and anterior temporal cortex activation in a moral judgment task-Preliminary functional MRI results in normal subjects. Arq Neurosiquiatr, 59, 657-664. doi:10.1590/S0004-282X2001000500001

Moll, J., Roland Z., R. de Oliveira-Souza, Bramati, I. E., \& Grafman, J. (2002). Functional networks in emotion moral and nonmoral social judgments. NeuroImage, 16, 696-703. doi:10.1006/nimg.2002.1118

Koenigs, M., Young, L., Adolphs, R., Tranel, D., Cushman, F., Hauser, M., et al. (2007a). Damage to the prefrontal cortex increases utilitarian moral judgments. Nature, 446, 908-911. doi:10.1038/nature05631

Koenigs, M., \& Tranel D. (2007b). Irational economic decision-making after ventromedial prefrontal damage: Evidence from the ultimatums game. Journal of Neuroscience, 27, 951-956. doi:10.1523/JNEUROSCI.4606-06.2007

Oschner, K. N., \& Lieberman, M. D. (2001). The emergence of social cognitive neuroscience. American Psychologist, 56, 717-734. doi:10.1037/0003-066X.56.9.717

Shen, W. B., \& Liu, C. (2010). Moral judgment: Rational processing or irrational processing?-A perspective from cognitive science. Journal of Psychological Science, 33, 807-810.

Spence, J. (1987). Centrifugal versus centripetal tendencies in psychology: Will the center hold? American Psychologist, 42, 1052 1054. doi:10.1037/0003-066X.42.12.1052

Watson J. D., \& Crick F. H. C. (1953). A structure for deoxyribose nucleic acid. Nature, 171, 737-738. 